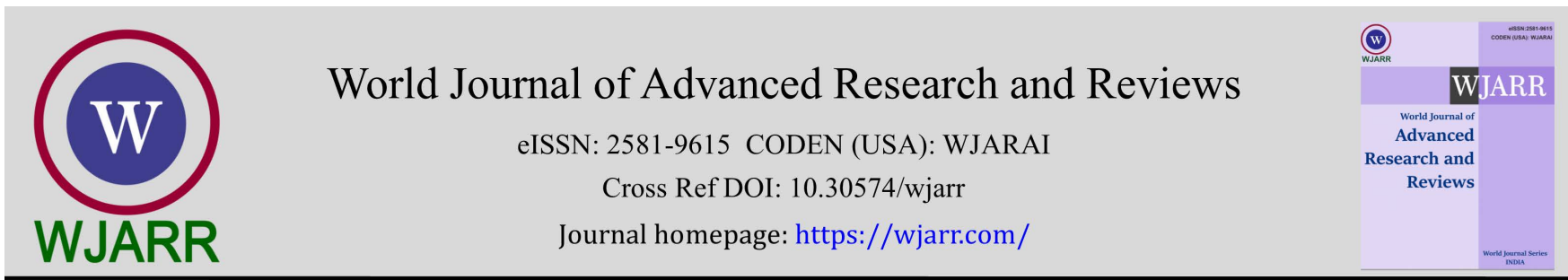

(CASE REPORT)

\title{
Severe leukocytosis and cytokine storm in a patient with covid-19 pneumonia
}

\author{
Iza David Zabaneh, Pamela K. Fonseca, Jennifer T. Prime and Sreedhara B. Alla * \\ Northwest Louisiana Nephrology Center, Shreveport, Louisiana, USA.
}

World Journal of Advanced Research and Reviews, 2021, 09(03), 215-217

Publication history: Received on 08 February 2021; revised on 10 March 2021; accepted on 13 March 2021

Article DOI: https://doi.org/10.30574/wjarr.2021.9.3.0098

\begin{abstract}
Since the emergence of the first cases of COVID-19 that was first reported in Wuhan China, the virus has spread globaly at a fast rate. It has carried a significant increase in the mortality rate. Most of these cases have been secondary to severe pneumonia as well as an increased incidence of thromboembolic complications leading to pulmonary embolism, myocardial infarctions, and strokes. It has been observed that many of these severe COVID-19 pneumonias have been associated with an increased levels of inflammatory markers including Cytokines and Interleukin 3 and 6 which can lead to an exaggereted humoral response with increased immunoglobulin production and tumor necrosis factors that can lead to lung tissue destruction. There is an overwhelming demand to find a mechanism to stop that vicious cycle and minimize tissue destruction. Thus, there is a need for more extensive studies regarding this medical dilemma in order to minimize the number of deaths around the world which so far has almost reached 2.5 million.
\end{abstract}

Keywords: Covid-19 Infection; Pneumonia; Cytokines; Interleukins; Tumor Necrosis Factor; Tocilizumab

\section{Introduction}

COVID-19 has been associated with severe mortality and morbidity leading to death. Pneumonia and hypoxia have been one of the major indicators of severe disease and carry poor prognostic signs. We herein report a case of a patient with chronic lymphocytic leukemia who developed severe pneumonia associated with cytokine storm leading to her demise.

\section{Case report}

A 64 years old African American lady with past medical history of type 2 diabetes mellitus non-insulin dependent, hypertension and stable asymptomatic chronic lymphocytic leukemia under observation by an oncologist, presented with an 8 days history of fatigue and intermittent fevers of 101 degrees Fahrenheit. She stayed at home hoping that her symptoms would resolve spontaneously. The morning prior to coming to the emergency room department, she woke up with inability to get out of bed, worsening dyspnea, and a fever of 100.6 degrees Fahrenheit. On arrival at the emergency room she had a fever of 103.5 and an oxygen saturation of $87 \%$ on room air. Blood pressure was $110 / 60$ with a pulse rate of 110 beats per minute. Physical examination revealed extensive crackles in both lung fields. She was also using her accessory muscles of respiration and was breathing around 22 times per minute. Chest radiograph showed extensive bilateral pulmonary alveolar airspace infiltrations suggestive of extensive bilateral pneumonia.

The rapid PCR test for SARS CoV-2 Coronavirus test using nasopharyngeal swab was positive. Both blood and sputum cultures were negative. Initial CBC was significant for severe leukocytosis with WBC count of 186 E3/ uL (normal range 3.7-10.6 E3/uL). Due to her CLL, her baseline WBC on her last visit to the oncologist a month prior to her illness was 54 E3/uL. Hemoglobin was $10.4 \mathrm{~g} / \mathrm{dl}$ with a platelet count of $109 \mathrm{E} 3 / \mathrm{uL}$. Other blood tests showed a blood urea nitrogen of $32 \mathrm{mg} / \mathrm{dl}$ and a creatinine of $1.13 \mathrm{mg} / \mathrm{dl}$. Patient had a potassium of $6.8 \mathrm{mmol} / \mathrm{L}$ (normal range $3.5-5.1 \mathrm{mmol} / \mathrm{L}$ ).

\footnotetext{
${ }^{*}$ Corresponding author: Sreedhara B. Alla

Northwest Louisiana Nephrology Center, Shreveport, Louisiana, USA.
}

Copyright (C) 2021 Author(s) retain the copyright of this article. This article is published under the terms of the Creative Commons Attribution Liscense 4.0. 
Plasma potassium level was $5.4 \mathrm{mmol} / \mathrm{L}$. Picture was felt to be due to pseudohyperkalemia. C-reactive protein was 75 $\mathrm{mg} / \mathrm{L}$ (normal range <= $10 \mathrm{mg} / \mathrm{L}$ ). Ferritin level 777ng/ml (normal range 11.1-264 ng/ml). Interleukin6 (IL-6) level was $10 \mathrm{pg} / \mathrm{mL}$ (normal range < 1.8pg/mL), Blood smear was consistent with significant leukocytosis with few atypical white blood cells compatible with the patient's history of CLL. The patient was admitted to the intensive care unit. Because of hypoxia, she required high-flow oxygen, placing her in a prone position. She received convalescent plasma, Actemra (Tocilizumab) as well as high dose steroids. WBC count peaked at $204 \mathrm{E} 3 / \mathrm{uL}$ ).

Despite above measures, the patient's condition continued to deteriorate with worsening hypoxia requiring intubation and mechanical ventilation. On the sixth day of admission, she developed cardiopulmonary arrest with unsuccessful resuscitation.

\section{Discussion}

COVID-19 has been associated with significant mortality and morbidity. According to the coronavirus resource center, as of end of February 2021, there were 2,528,535 total deaths worldwide, with cases of death exceeding half a million in the United States of America. One of the major causes of death has been attributed to severe lung injury and respiratory failure. The virus has been connected to its ability to molecularly trigger an acute inflammatory process through its molecular mimicry, triggering multi-organ damage [1]. One of the mechanisms of injury related to COVID19 was felt to be due to activation of Cytokine storm [2,3] This process has been observed with a number of conditions including sepsis [4], viral infections [5] Similar to many severe diseases, COVID-19 has been associated with an increased level of Interleukin 6. This is a polypeptide cytokine which is produced by T cells. This activates the production of humoral response B cells to produce the immunoglobulins IgG, IgM and IgA. It does work synergistically with Interleukin 3 (IL-3) [6]. In addition, Interleukin 1 (TNF tumor necrosis factor) also gets activated in this process. Such exaggerated responses to COVID -19 act as pleiotropic chemotactic to neutrophils, lymphocytes and macrophages that would attack the inflamed sites, which are usually the lung tissue leading to cell necrosis and cytolysis [7, 8]. One of the possible approaches to treat high level interleukin 6 Covid-19 pneumonia is utilizing Tocilizumab an Interleukin 6 inhibitor as a potential approach to cytokine storm [9]. However, several studies have been inconclusive with further studies needed to evaluate its efficacy.

In this case, we believe that the patient had a cytokine storm, causing leukemoid reaction in a patient with underlying chronic lymphocytic leukemia, with elevated inflammatory markers and clinical presentation supporting this diagnosis. Of interest, the patient had pseudohyperkalemia due to the severe leukocytic response to the virus which was confirmed by doing both serum and plasma potassium simultaneously. More clinical and laboratory research is needed to evaluate the inflammatory markers associated with COVID-19 cytokine storm, and hopefully find a mechanism to abort such a storm in the future.

\section{Conclusion}

In this case, we believe that the patient had a cytokine storm, causing leukemoid reaction in a patient with underlying chronic lymphocytic leukemia, with elevated inflammatory markers and clinical presentation supporting this diagnosis. Of interest, the patient had pseudohyperkalemia due to the severe leukocytic response to the virus which was confirmed by doing both serum and plasma potassium simultaneously. More clinical and laboratory research is needed to evaluate the inflammatory markers associated with COVID-19 cytokine storm, and hopefully find a mechanism to abort such a storm in the future.

\section{Compliance with ethical standards}

\section{Acknowledgments}

The group acknowledges Dr. Alla for his oversight of the published paper.

\section{Disclosure of conflict of interest}

There was no conflict of interest among corresponding authors.

\section{Statement of informed consent}

Proper informed consents were obtained. 


\section{References}

[1] Francesca Angileri, Sébastien Legare, Antonella Marino Gammazza, Everly Conway de Macario, Alberto JL Macario, Francesco Cappello. Molecular Mimicry May Explain Multi-Organ Damage in Covid-19. Autoimmunity Reviews. 2020.

[2] David C. Fajgenbaum, Carl H. June. Cytokine Storm. N Engl J Med. 2020; 383: 2255-2273.

[3] Dipak Panigrahy, Molly M. Gilligan, Sui Huang, Allison Gartung, Irene Cortés-Puch, Patricia J. Sime, Richard P. Phipps, Charles N. Serhan, Bruce D. Hammock. Inflammation Resolution: A Dual -Prolonged Approach to Averting Cytokine Storms in COVID-19? Cancer and Metastasis Reviews. 2020; 39(2): 337-340.

[4] Georg F Weber. Interleukin -3 amplifies acute inflammation and is a potential therapeutic target in sepsis. Science13 Mar 2015; 347(6227): 1260-1265.

[5] Natalia Hagau, Adriana Slavcovici, Daniel N Gonganau, Simona Oltean, Dan S. Diruz, Erika S. Brezoszki, Mihaela Maxim, Constantin Ciuce, Monica Mlesnite, Rodica L. Gavrus, Carmen Laslo, Radu Hagau, Magda Petrescu, Daniela M. Studnicska. Clinical aspects and cytokine response in severe H1N1 Influenza A virus Infection. Critical Care. 2010; 14(6): 1-10.

[6] Mohammed A Omair, Interleukin 6. Rheumatology. 2015; 1: 485-491.

[7] Jennifer R Tisonick, Marcus J Korth, Cameron P Simmons, Jeremy Farrar, Thomas R Martin, Michael G Katze. Into the EYE of The Cytokine Storm. Microbiol Mol Biol Rev. 2012 Mar; 76(1): 16-32.

[8] Alessandro Rubini. Interleukin-6 and lung inflammation: evidence for a causative role in inducing respiratory system resistance increments. Inflamm Allergy Drug Targets. 2013 Oct.

[9] Ivan O. Rosas, Norbert Bräu, Michael Waters, Ronaldo C. Go, Bradley D. Hunter, Sanjay Bhagani, Daniel Skiest, Mariam S. Aziz, Nichola Cooper, Ivor S. Douglas, Sinisa Savic, Taryn Youngstein, Lorenzo Del Sorbo, Antonio Cubillo Gracian, David J. De La Zerda, Andrew Ustianowski, Min Bao, Sophie Dimonaco, Emily Graham, Balpreet Matharu, Helen Spotswood, Larry Tsai, Atul Malhotra. Tocilizumab in Hospitalized Patients with severe Covid-19 pneumonia. NEJM. Feb 25, 2021. 